\title{
DNA methylation is involved in the aberrant expression of miR-133b in colorectal cancer cells
}

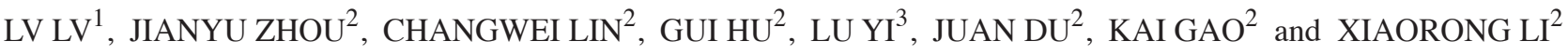 \\ Departments of ${ }^{1}$ Emergency Medicine, ${ }^{2}$ General Surgery and ${ }^{3}$ Neurosurgery, The Third Xiangya Hospital, \\ Central South University, Changsha, Hunan 410013, P.R. China
}

Received September 23, 2014; Accepted April 30, 2015

DOI: $10.3892 / \mathrm{ol} .2015 .3336$

\begin{abstract}
The dysregulation of microRNA (miRNA) expression is highly involved in cancer. Recently, a number of studies have demonstrated that the silencing of specific miRNAs is associated with DNA methylation. The muscle-specific miRNA-113b (miR-133b) is markedly downregulated in human colorectal cancer (CRC) compared with healthy colon cells, and is critical in the regulation of CRC cell proliferation and apoptosis. However, the mechanism of miR-133b downregulation in CRC has yet to be elucidated. Therefore, the aim of the present study was to determine the existence of an association between DNA methylation and miR-133b expression in CRC cells. It was identified that miR-133b promoter hypermethylation is upregulated in CRC tissues. To investigate the role of miR-133b methylation in CRC cells, the survival, cell cycle and invasion were analyzed in HT-29 and SW620 CRC cells treated with 5-aza-2'-deoxycytidine (5-Aza-CdR), 4-phenylbutyric acid (PBA) and 5-Aza-CdR/PBA. Functional analysis demonstrated that demethylation increased the expression of miR-133b, which restored migration and apoptosis in CRC cells. Thus, these results indicate that the regulation of miR-133b methylation may provide a novel therapeutic strategy for CRC treatment.
\end{abstract}

\section{Introduction}

Colorectal cancer (CRC) is the third most commonly diagnosed type of cancer worldwide and demonstrates a high incidence and mortality rate (1), with the number of cases continuing to rise, particularly in Asian countries (2). Despite a progressive improvement in the available treatment regimens, prolonging patient survival following surgery continues to be predominantly hindered by post-operative recurrence and metastasis. Therefore, the development of more efficient

Correspondence to: Dr Xiaorong Li, Department of General Surgery, The Third Xiangya Hospital of Central South University, 138 Tongzipo Road, Changsha, Hunan 410013, P.R. China

E-mail: lixiaorong@medmail.com.cn

Key words: colorectal carcinoma, DNA methylation, microRNA-133b treatment regimens and greater understanding of the precise molecular mechanisms of CRC are required.

MicroRNAs (miRNAs) are conserved short sequences of non-coding RNA that are 22-24 nucleotides long and appear to be involved in a broad range of biological functions, such as cell invasion, metastasis, proliferation, angiogenesis and apoptosis $(3,4)$. miRNAs bind to complementary target mRNA sequences and regulate the expression of target genes at the post-transcriptional level. In the last decade, an increasing number of studies have identified miRNAs as critical regulators of carcinogenesis and tumor progression, affecting tumor growth, progression, metastasis and drug resistance (5-7). As a result, an increasing number of studies have demonstrated the potential of miRNAs as novel therapeutic targets and diagnostic markers $(8,9)$.

Despite a lack of comprehensive understanding of the mechanisms underlying miRNA dysregulation in cancer, a number of studies have demonstrated that the silencing of specific miRNAs and DNA methylation are strongly associated. For example, in cancer cells, hypermethylated $\mathrm{CpG}$ islands are found within the DNA sequences encoding miR-127 and miR-124a. Furthermore, miR-127 expression was restored in a human bladder cancer cell line following treatment with chromatin-modifying agents $(10,11)$ and restoration of miR-124a expression was observed in a CRC cell line following genetic disruption of DNA methyltransferases (11). Similarly, miR-34b and miR-34c CpG island hypermethylation was observed in CRC $(12,13)$. Thus, the silencing of miRNA expression in cancer appears to be largely mediated by DNA methylation, particularly the methylation of tumor suppressor genes.

miR-133b is known to be a muscle-specific miRNA and has been reported to be involved in the development of skeletal muscle (14). However, previous studies have supported a broader expression pattern of miR-133b in a diverse range of tissues. Furthermore, miR-133b has previously been identified as a tumor suppressor, with miR-133b deregulation observed in colorectal, head and neck, squamous cell, lung, and bladder cancers (15-17). miR-133b regulates various target genes in these cancer types, such as myeloid cell leukemia sequence 1, B-cell lymphoma-like 2, fascin homolog 1, c-Met and epidermal growth factor receptor $(16,18-21)$. Although previous studies have demonstrated that miR-133b is significantly downregulated in CRC, the epigenetic mechanisms associated with aberrant miR-133b expression in CRC remain unclear. Therefore, the 
present study aimed to determine the epigenetic mechanisms involved in the regulation of miR-133b silencing in CRC cells.

\section{Materials and methods}

Cell lines and specimens. Five CRC cell lines, consisting of the HT-29, HCT-116, SW480, SW620 and LoVo cell lines (American Type Culture Collection, Rockville, MD, USA), and the healthy human colonic epithelial HCoEpiC cell line (Sciencell Research Laboratories, Carlsbad, CA, USA) were used in the present study. The cells were maintained under the recommended conditions.

In total, 6 human CRC, 2 adjacent non-tumor and 2 healthy colorectal tissue samples were obtained in March 2014 from patients that were diagnosed with colon adenocarcinoma at the Department of General Surgery, The Third Xiangya Hospital of Central South University (Changsha, Hunan, China). The specimens were obtained subsequent to surgical resection and were immediately frozen at $-80^{\circ} \mathrm{C}$ until use. Written informed consent was obtained from all patients. The methodology used in the present study conformed to the standards set by the Declaration of Helsinki (22) and was approved by the ethics committee of The Third Xiangya Hospital of Central South University.

5-aza-2'-deoxycytidine (5-Aza-CdR) and 4-phenylbutyric acid (PBA) treatment. To evaluate the expression of miR-133b, all cell lines were treated with $3 \mu \mathrm{M}$ of the demethylation agent 5-Aza-CdR, $3 \mathrm{mM}$ histone deacetylase (HDAC) inhibitor (PBA), or $3 \mu \mathrm{M}$ 5-Aza-CdR (Sigma-Aldrich, St. Louis, MO, USA) and $3 \mathrm{mM}$ PBA (Sigma-Aldrich) for 24, 48 and $72 \mathrm{~h}$. The cells were seeded $24 \mathrm{~h}$ prior to treatment.

$R N A$ extraction and reverse transcription quantitative-polymerase chain reaction ( $R T-q P C R)$. Total RNA was extracted from the CRC cell lines and HCoEpiC cells using TRIzol reagent (Invitrogen Life Technologies, Carlsbad, CA, USA) and then reverse-transcribed into complementary DNA. HmiRQP0167 primers were obtained from GeneCopoeia, Inc. (Rockville, MD, USA). The PCR protocol consisted of 35 cycles at $95^{\circ} \mathrm{C}$ for $3 \mathrm{~min}, 95^{\circ} \mathrm{C}$ for $12 \mathrm{sec}$ and $58^{\circ} \mathrm{C}$ for $30 \mathrm{sec}$. The relative expression level of miRNA was normalized to the expression of the internal control U6 small nuclear RNA using the $2^{-\Delta \Delta C t}$ method (23).

Methylation-specific PCR (MSP). MSP was used to analyze the methylation status of healthy and CRC tissues, as well as healthy, HT-29 and SW620 cell lines, as described previously (24). Genomic DNA was extracted from tissues or cells using the DNA Extraction Mini Kit (Qiagen $\mathrm{GmbH}$, Hilden, Germany) according to the manufacturer's instructions. Extracted DNA samples underwent sodium bisulfite modification using a Universal CpGenome ${ }^{\mathrm{TM}}$ DNA Modification Kit (Merck Millipore, Darmstadt, Germany). MethPrimer software (available from www.methdb.de) was used to design the two sets of primers, as follows: methylated miR-133b forward, 3'-TTTATTTAAAATATAAAAATA GCGG-5' and reverse, 3'-TCACCCAAACTAAAATACAAT AACG-5'; and unmethylated miR-133b forward, 3'-TTTTTA TTTAAAATATAAAAATAGTGG-5' and reverse, 3'-ACC
CAAACTAAAATACAATAACACT-5'. MSP was performed using the previously described negative controls and conditions (25).

Combined bisulfite restriction analysis (COBRA). The methylation pattern of miR-133b was analyzed using COBRA, as previously described (26). Briefly, the process involved sodium bisulfite treatment, which was followed by PCR, restriction digestion and quantitation (27). The following primers were designed using MethPrimer software to specifically amplify the methylated sequence of miR-133b: Forward, 3'-GTATTT AGTATAAAGAAAGTAA-5' and reverse, 3'-ACCAATACC CATAAACAACG-5'.

Cell viability assay. The cells were seeded into 96-well plates at a density of $1 \times 10^{3}$ cells/well. Following treatment, cell viability was evaluated at $0,24,48$ and $72 \mathrm{~h}$ using a Cell Counting Kit-8 (CCK-8; Dojindo Laboratories, Kumamoto, Japan), according to the manufacturer's instructions. Optical density was measured at a wavelength of $450 \mathrm{~nm}$ and all data is based on the results of three independent experiments with six replicates.

Transwell migration assay. Transwell migration chambers (BioCoat Matrigel Invasion Chamber; BD Biosciences, Franklin Lakes, NJ, USA) were used to evaluate cell invasion. A total of $2.5 \times 10^{5}$ cells/well were starved in serum-free medium (Invitrogen Life Technologies) for $24 \mathrm{~h}$, then resuspended in serum-free medium. The cells were added to the upper chamber, while the lower chamber was filled with basic medium containing $10 \%$ fetal bovine serum. Following incubation for $24 \mathrm{~h}$, the cells that had migrated across the membrane were fixed with $75 \%$ alcohol and stained with crystal violet (Amresco LLC, Solon, OH, USA) for 20 min. Migrated cells were then counted using an inverted microscope (TS100; Nikon Corporation, Tokyo, Japan). All experiments were repeated in triplicate.

Flow cytometry analysis of the cell cycle. Flow cytometry was used to analyze cell cycle distribution, as previously described (27). Briefly, 5-Aza-CdR or PBA-treated cells were fixed in ice-cold $70 \%$ ethanol and stained with propidium iodide. The cell cycle profiles were assayed using EPICS Elite ESP flow cytometry (Beckman Coulter, Inc., Brea, CA, USA) at a wavelength of $488 \mathrm{~nm}$ and the data were analyzed using the BD CellQuest software (BD Biosciences, San Jose, CA, USA).

Statistical analysis. Data in the present study are expressed as the mean \pm standard deviation of a minimum of three independent experiments, unless otherwise stated. Analyses were conducted using SPSS software version 17.0 (SPSS, Inc., Chicago, IL, USA). An unpaired Student's $t$-test was used for comparisons between two groups. $\mathrm{P}<0.05$ was considered to indicate a statistically significant difference.

\section{Results}

Reduced expression of miR-133b in CRC. The expression of miR-133b was previously reported to be decreased in CRC 

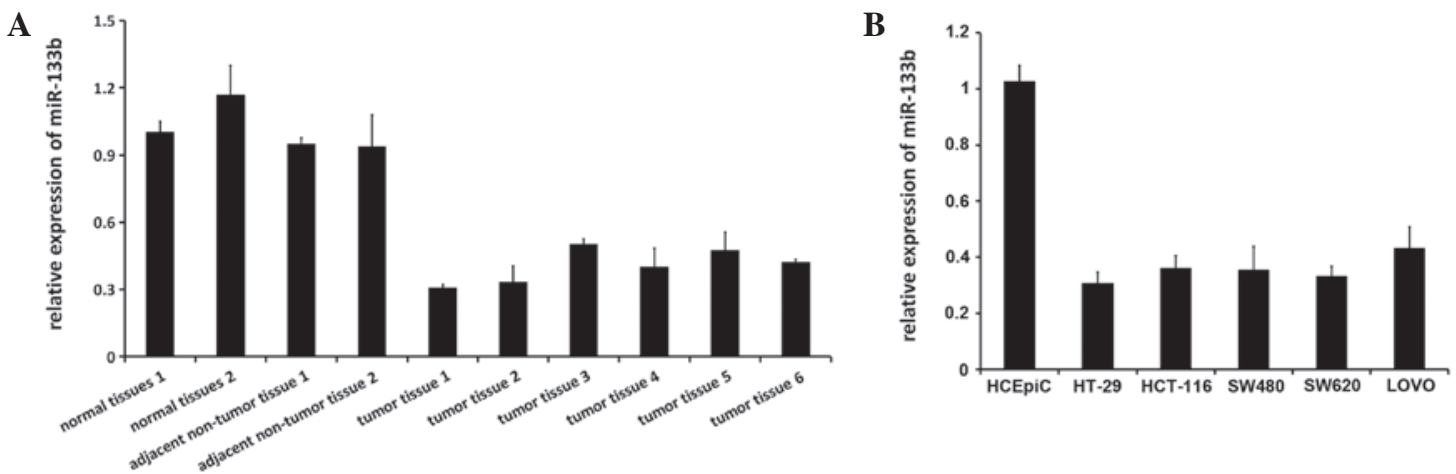

Figure 1. miR-133b expression is downregulated in CRC tissues and cell lines. (A) Reverse transcription-quantitative polymerase chain reaction identified significant downregulation in the expression levels of miR-133b in CRC tissues but not in healthy or matched adjacent non-tumor tissues. U6 small nuclear RNA was used as the endogenous control. (B) Relative expression level of miR-133b in one healthy colon cell line and five CRC cell lines. CRC, colorectal cancer; miR, microRNA.

A

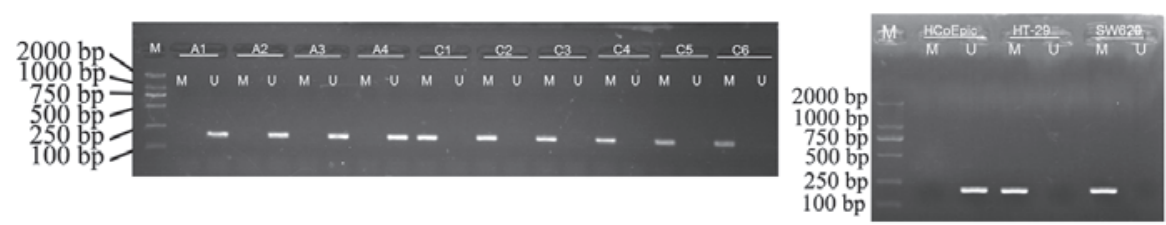

B

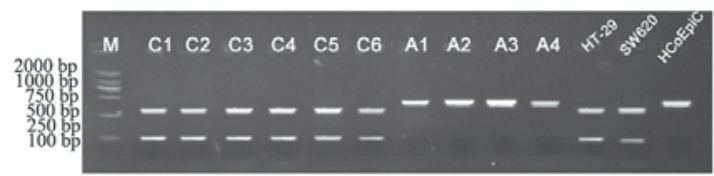

C

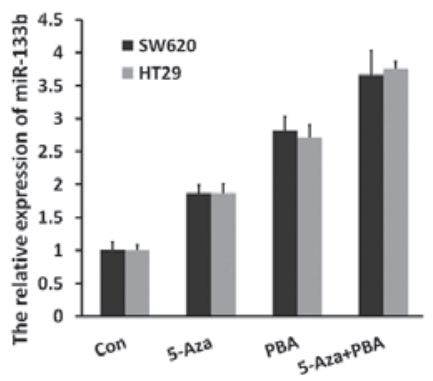

$\mathbf{E}$

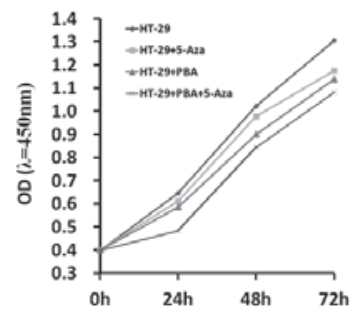

D
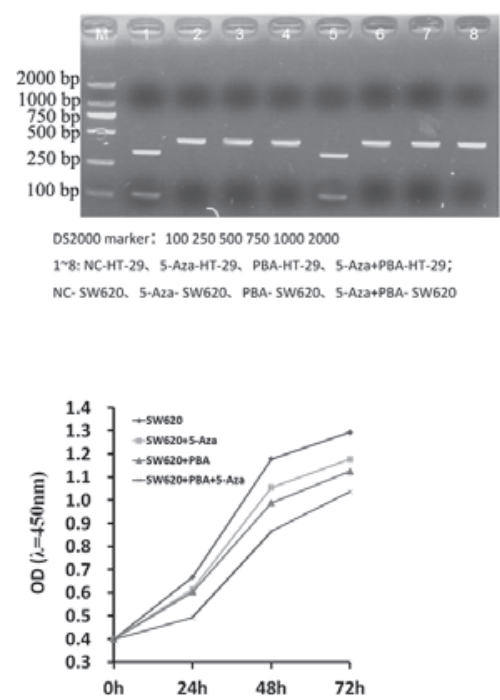

Figure 2. miR-133b methylation in CRC tissue samples and cell lines, and reactivation of miR-133b by demethylation. (A and B) Methylation-specific PCR, revealing frequent methylation of miR-133b in CRC tissue samples and the CRC HT-29 and SW60 cell lines, and healthy human colonic HCoEpiC cell lines. A1-A2, Healthy colon tissue; A3-A4, adjacent non-tumor tissue; C1-C6, human CRC tissue. (C) Reverse transcription-quantitative PCR and (D) combined bisulfite restriction analysis demonstrating that pharmacological demethylation can restore miR-133b expression in CRC cells. (E) Survival curve of CRC cells following treatment with 5-Aza/PBA, determined by using CCK-8. CRC, colorectal cancer; PCR, polymerase chain reaction; M, methylated; U, unmethylated; miR, microRNA; con, control; 5-Aza, 5-aza-2'-deoxycytidine; PBA, 4-phenylbutyric acid; OD, optical density; CCK-8, cell counting kit-8.

tissues and two colon adenocarcinoma cell lines (21). Therefore, the present study used RT-qPCR to determine miR-133b expression levels in healthy colon tissues, human CRC tissue and adjacent non-tumor tissue, as well as five CRC cell lines, consisting of the HT-29, HCT-116, SW480, SW620 and
LOVO cell lines, and the healthy HCoEpiC colon cell line. miR-133b expression was notably downregulated or silenced in all the investigated CRC tissues and cell lines $(\mathrm{P}=0.013)$, but miR-133b was broadly expressed in all healthy tissues and HCoEpiC cell lines (Fig. 1A and B). 
A

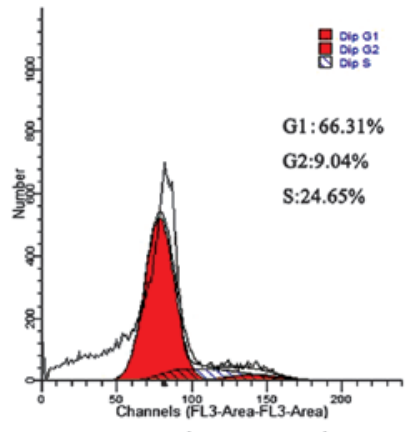

D

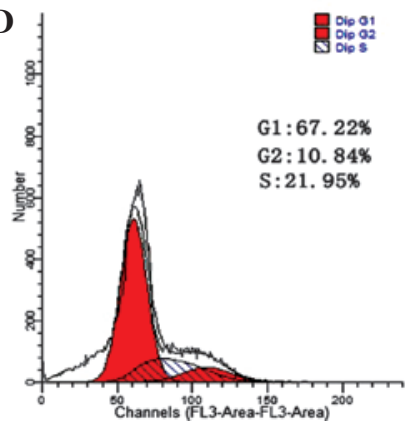

B

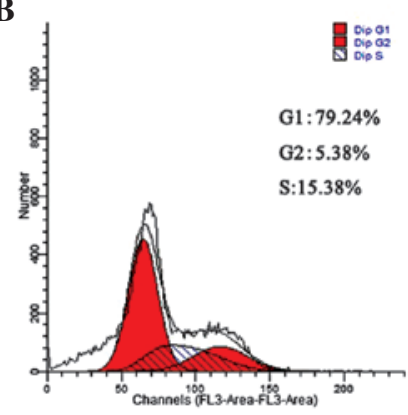

$\mathbf{E}$

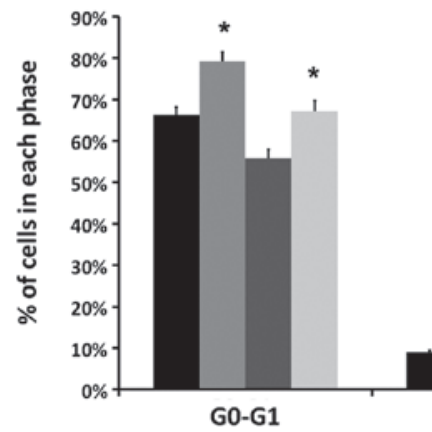

C

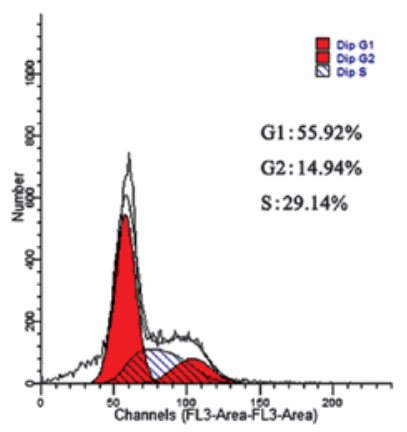

- HT-29

- HT-29+5-Aza+PBA

- SW620

SW620+5-Aza+PBA

Figure 3. Effect of miR-133b demethylation on the cell cycle distribution of CRC HT-29 and SW620 cells. Representative cell cycle analysis graphs of (A) control HT-29 cells, (B) HT-29 5-Aza/PBA-treated cells, (C) control SW620 cells and (D) SW620 5-Aza/PBA-treated cells. (E) Summarized flow cytometry data. Results are presented as the mean \pm standard deviation and are based on three independent experiments. ${ }^{*} \mathrm{P}<0.05$ vs. untreated control cells, determined using a Student's $t$-test. miR, microRNA; 5-Aza, 5-aza-2'-deoxycytidine; PBA, 4-phenylbutyric acid.
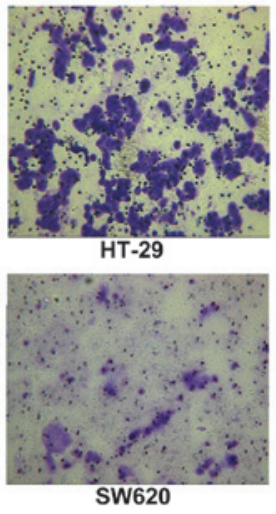

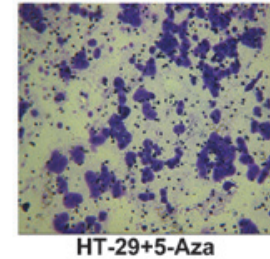

HT-29+5-Aza

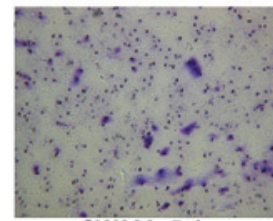

SW620+5-Aza

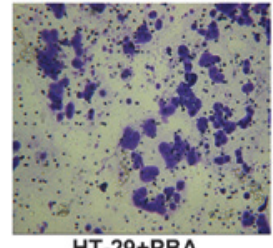

HT-29+PBA

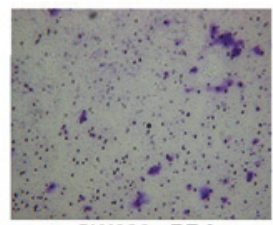

SW620+PBA

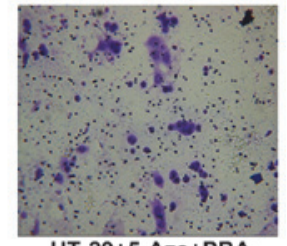

HT-29+5-Aza+PBA

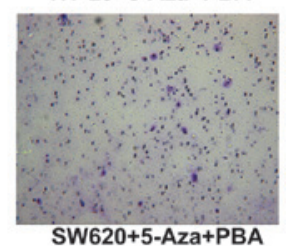

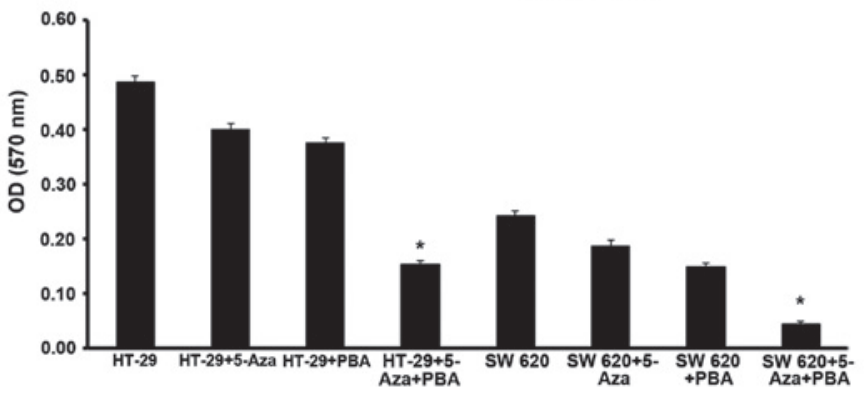

Figure 4. miR-133b demethylation inhibits tumor cell invasion. Colorectal cancer HT-29 and SW620 cells were treated with 5-Aza, PBA and 5-Aza/PBA. The number of invading tumor cells was calculated by analyzing Transwell invasion assays, and the miR-133b demethylation and control groups were compared. ${ }^{*} \mathrm{P}<0.05$ vs. untreated control cells. miR, microRNA; 5-Aza, 5-aza-2'-deoxycytidine; PBA, 4-phenylbutyric acid; OD, optical density.

Promoter methylation of miR-133b and restoration of miR-133b expression by demethylation. The present study investigated whether miR-133b silencing in CRC was caused by CpG methylation in the promoter region. MSP analysis revealed that miR-133b was frequently methylated in the investigated CRC tissues and in the SW260 and HT-29 colon cancer cell lines, and was strongly associated with expression levels. However, no miR-133b methylation was identified in the healthy colon tissue samples or healthy colon cell line (Fig. 2A and B). This finding was supported by results obtained from the RT-qPCR and COBRA analyses (Fig. 2C and D), which indicated that the expression of miR133b was 
increased in SW620 and HT-29 cells treated with Aza, PBA and Aza+PBA. CCK-8 was also used to assess the effects of demethylation agents on SW620 and HT-29 cell proliferation. It was observed that inhibition of proliferation was induced by Aza or PBA treatment, whilst a significantly higher level of inhibition was induced by Aza+PBA treatment (Fig. 2E).

miR-133b demethylation affects cell cycle arrest and cell invasion in CRC. The present study investigated the effects of miR-133b expression on the cell cycle and invasive ability of the HT-29 and SW620 CRC cell lines. Representative results of cell cycle distribution in control and 5-Aza-CdR/PBA-treated cells are shown in Fig. 3A-D. Flow cytometry analysis revealed a statistically significant increase in the number of 5 -Aza-CdR/PBA-treated cells in the G0/G1 phase (13\% in HT-29 cells and 12\% in SW620 cells) compared with the number of control cells in the G0/G1 phase $(\mathrm{P}<0.05)$. This was accompanied by a significant decrease in the number of 5-Aza-CdR/PBA-treated cells in the S and G2-M phase compared with the control cells $(\mathrm{P}=0.027$; Fig. 3E).

The effect of miR-133b methylation on tumor metastasis was evaluated by performing a cell invasion assay. Analysis of the Transwell invasion chamber assay data indicated that the invasive ability of cells in the miR-133b demethylation group treated with 5-Aza-CdR/PBA was significantly inhibited compared with the control group $(\mathrm{P}<0.05$; Fig. 4).

The aforementioned results indicate that miR-133b methylation reduced $\mathrm{CRC}$ cell cycle progression and the invasiveness of CRC tumor cells.

\section{Discussion}

In previous years, an increasing number of studies have identified the dysregulation of miRNA expression in a broad range of cancer types $(3,5,6,8,11,12)$. Epigenetic mechanisms were found to be the key mediators underlying the downregulation of miRNA expression. For example, miR-126 was downregulated in human cancer cells and was activated by inhibitors of DNA methylation and histone deacetylation (28). In addition, the activation of miR-512-5p by epigenetic treatment resulted in the apoptosis of gastric cancer cells (29). Similarly, miR-34b and miR-34c are novel tumor suppressors that are frequently silenced by DNA methylation in gastric cancer (12). In addition, the hypermethylation of miRNA genes that is frequently observed in CRC, as well as the association between the clinicopathological features and methylation status, provides evidence for the hypothesis that epigenetic instability is an important event in tumorigenesis, particularly in CRC. The present findings revealed miRNA methylation is involved in an epigenetic field defect and may be a target of epigenetic therapy in cancer.

Considering the high occurrence, mortality and relapse rates of CRC, there is an acute requirement for the identification of novel prognostic factors and development of therapeutic strategies. miR-133b is known to be a muscle-specific miRNA (30) and has recently been reported to act as a tumor suppressor that is dysregulated in colorectal, head and neck, squamous cell, lung, and bladder cancer (20,31). Aberrant miR-133b expression has been identified in CRC cancer tissues (24), with the overexpression of miR-133b in CRC cells observed to induce apoptosis and G1 phase cell cycle arrest (20). However, the epigenetic mechanisms associated with aberrant miR-133b expression remain unclear.

The present study identified that the expression of miR-133b was significantly decreased in CRC cells with miR-133b promoter hypermethylation, indicating that miR-133b was silenced by epigenetic mechanisms. Furthermore, inhibitors of DNA methylation and HDAC reduced the extent of DNA methylation and activated the expression of silenced miR-133b in human CRC cells. In addition, functional studies have revealed that miR-133b methylation interfered with CRC cell apoptosis, cell cycle progression and cell invasion. The results of the present study are consistent with those of previous studies which revealed that miR-133b inhibits cell growth and invasion in bladder (32), non-small-cell lung (33) and prostate (34) cancer.

In conclusion, the present study observed a high frequency of miR-133b hypermethylation in CRC cells and tissue samples, indicating that methylation of the miR-133b gene may be a useful diagnostic marker for patients with CRC. However, future in vivo studies are required to investigate the tumor-suppressive role of miR-133b in CRC.

\section{References}

1. Jemal A, Bray F, Center MM, Ferlay J, Ward E and Forman D: Global cancer statistics. CA Cancer J Clin 61: 69-90, 2011.

2. Sung JJ, Lau JY, Goh KL and Leung WK; Asia Pacific Working Group on Colorectal Cancer: Increasing incidence of colorectal cancer in Asia: Implications for screening. Lancet Oncol 6: 871-876, 2005.

3. Calin GA and Croce CM: MicroRNA signatures in human cancers. Nat Rev Cancer 6: 857-866, 2006.

4. Bartel DP: MicroRNAs: Genomics, biogenesis, mechanism and function. Cell 116: 281-297, 2004.

5. Lu J, Getz G, Miska EA, et al: MicroRNA expression profiles classify human cancers. Nature 435: 834-838, 2005.

6. Esquela-Kerscher A and Slack FJ: Oncomirs-microRNAs with a role in cancer. Nat Rev Cancer 6: 259-269, 2006.

7. Manikandan J, Aarthi JJ, Kumar SD and Pushparaj PN: Oncomirs: The potential role of non-coding microRNAs in understanding cancer. Bioinformation 2: 330-334, 2008.

8. Meltzer PS: Cancer genomics: Small RNAs with big impacts. Nature 435: 745-746, 2005.

9. Fang K, Qian F and Chen YQ: MicroRNAs as regulators in normal hematopoietic and leukemia stem cells: Current concepts and clinical implications. Curr Mol Med 12: 536-546, 2012.

10. Saito Y, Liang G, Egger G, et al: Specific activation of microRNA-127 with downregulation of the proto-oncogene BCL6 by chromatin-modifying drugs in human cancer cells. Cancer Cell 9: 435-443, 2006.

11. Lujambio A, Ropero S, Ballestar E, et al: Genetic unmasking of an epigenetically silenced microRNA in human cancer cells. Cancer Res 67: 1424-1429, 2007.

12. Suzuki H, Yamamoto E, Nojima M, et al: Methylation-associated silencing of microRNA-34b/c in gastric cancer and its involvement in an epigenetic field defect. Carcinogenesis 31: 2066-2073, 2010.

13. Toyota M, Suzuki H, Sasaki Y, et al: Epigenetic silencing of microRNA-34b/c and B-cell translocation gene 4 is associated with CpG island methylation in colorectal cancer. Cancer Res 68: 4123-4132, 2008.

14. Koutsoulidou A, Mastroyiannopoulos NP, Furling D, Uney JB and Phylactou LA: Expression of miR-1, miR-133a, miR-133b and miR-206 increases during development of human skeletal muscle. BMC Dev Biol 11: 34, 2011.

15. Wu J, Yang T, Li X, et al: Alteration of serum miR-206 and miR-133b is associated with lung carcinogenesis induced by 4-(methylnitrosamino)-1-(3-pyridyl)-1-butanone. Toxicol Appl Pharmacol 267: 238-246, 2013.

16. Yamamoto H, Kohashi K, Fujita A and Oda Y: Fascin-1 overexpression and miR-133b downregulation in the progression of gastrointestinal stromal tumor. Mod Pathol 26: 563-571, 2013. 
17. Zhao H, Li M, Li L, Yang X, Lan G and Zhang Y: MiR-133b is down-regulated in human osteosarcoma and inhibits osteosarcoma cells proliferation, migration and invasion and promotes apoptosis. PLoS One 8: e83571, 2013.

18. Crawford M, Batte K, Yu L, et al: MicroRNA 133B targets pro-survival molecules MCL-1 and BCL2L2 in lung cancer. Biochem Biophys Res Commun 388: 483-489, 2009.

19. Patron JP, Fendler A, Bild M, et al: MiR-133b targets antiapoptotic genes and enhances death receptor-induced apoptosis. PLoS One 7: e35345, 2012.

20. Hu G, Chen D, Li X, Yang K, Wang H and Wu W: miR-133b regulates the MET proto-oncogene and inhibits the growth of colorectal cancer cells in vitro and in vivo. Cancer Biol Ther 10: 190-197, 2010.

21. Xiang KM and Li XR: MiR-133b acts as a tumor suppressor and negatively regulates TBPL1 in colorectal cancer cells. Asian Pac J Cancer Prev 15: 3767-3772, 2014.

22. General Assembly of the World Medical Association: World Medical Association Declaration of Helsinki: Ethical principles for medical research involving human subjects. J Am Coll Dent 81: 14-18, 2014.

23. Izutsu N, Maesawa C, Shibazaki M, et al: Epigenetic modification is involved in aberrant expression of class III beta-tubulin, TUBB3, in ovarian cancer cells. Int J Oncol 32: 1227-1235, 2008.

24. Bandrés E, Cubedo E, Agirre X, et al: Identification by Real-time PCR of 13 mature microRNAs differentially expressed in colorectal cancer and non-tumoral tissues. Mol Cancer 5: 29, 2006.

25. Ogasawara S, Maesawa C, Yamamoto M, et al: Disruption of cell-type-specific methylation at the Maspin gene promoter is frequently involved in undifferentiated thyroid cancers. Oncogene 23: 1117-1124, 2004.
26. Karpinski P, Szmida E, Misiak B, et al: Assessment of three epigenotypes in colorectal cancer by combined bisulfite restriction analysis. Mol Carcinog 51: 1003-1008, 2012.

27. Xiang T, Li L, Yin X, et al: The ubiquitin peptidase UCHL1 induces G0/G1 cell cycle arrest and apoptosis through stabilizing p53 and is frequently silenced in breast cancer. PLoS One 7: e29783, 2012.

28. Saito Y, Friedman JM, Chihara Y, Egger G, Chuang JC and Liang G: Epigenetic therapy upregulates the tumor suppressor microRNA-126 and its host gene EGFL7 in human cancer cells. Biochem Biophys Res Commun 379: 726-731, 2009.

29. Saito Y, Suzuki H, Tsugawa H, et al: Chromatin remodeling at Alu repeats by epigenetic treatment activates silenced microRNA-512-5p with downregulation of Mcl-1 in human gastric cancer cells. Oncogene 28: 2738-2744, 2009.

30. Duan FT, Qian F, Fang K, et al: miR-133b, a muscle-specific microRNA, is a novel prognostic marker that participates in the progression of human colorectal cancer via regulation of CXCR4 expression. Mol Cancer 12: 164, 2013.

31. Wen D, Li S, Ji F, et al: miR-133b acts as a tumor suppressor and negatively regulates FGFR1 in gastric cancer. Tumour Biol 34 793-803, 2013.

32. Zhou Y, Wu D, Tao J, Qu P, Zhou Z and Hou J: MicroRNA-133 inhibits cell proliferation, migration and invasion by targeting epidermal growth factor receptor and its downstream effector proteins in bladder cancer. Scand J Urol 47: 423-432, 2013.

33. Liu L, Shao X, Gao W, Zhang Z, Liu P, Wang R, Huang P, Yin Y and Shu Y: MicroRNA-133b inhibits the growth of non-small-cell lung cancer by targeting the epidermal growth factor receptor. FEBS J 279: 3800-3812, 2012

34. Tao J, Wu D, Xu B, Qian W, Li P, Lu Q, Yin C and Zhang W: microRNA-133 inhibits cell proliferation, migration and invasion in prostate cancer cells by targeting the epidermal growth factor receptor. Oncol Rep 27: 1967-1975, 2012. 